\title{
Combined Information from Raman Spectroscopy and Optical Coherence Tomography for Enhanced Diagnostic Accuracy in Tissue Discrimination
}

\author{
Praveen C. Ashok ${ }^{1}$, Bavishna B. Praveen ${ }^{1}$, Nicola Bellini ${ }^{1}$, Andrew Riches ${ }^{2}$, Kishan Dholakia ${ }^{1}$, C. \\ Simon Herrington 2,3 \\ ${ }^{1}$ SUPA, School of Physics \& Astronomy, North Haugh, University of St Andrews, St Andrews, \\ Fife, Scotland, UK, KY16 9SS \\ ${ }^{2}$ School of Medicine, Medical and Biological Sciences Building, University of St Andrews, North \\ Haugh, St Andrews, Fife, Scotland, UK, KY16 9TF \\ ${ }^{3}$ Jacqui Wood Cancer Centre, University of Dundee, Ninewells Hospital, Dundee, Scotland, UK, \\ DD1 9SY
}

Keywords: Raman spectroscopy, Optical Coherence Tomography, Optical biopsy, Cancer detection, Multivariate analysis, Colon cancer, Multimodality, Texture analysis

\begin{abstract}
Optical spectroscopy and imaging methods have proved to have potential to discriminate between normal and abnormal tissue types through minimally invasive procedures. Raman spectroscopy and Optical Coherence Tomography (OCT) provides chemical and morphological information of tissues respectively, which are complementary to each other. When used individually they might not be able to obtain high enough sensitivity and specificity that is clinically relevant. In this study we combined Raman spectroscopy information with information obtained from OCT to enhance the sensitivity and specificity in discriminating between Colonic Adenocarcinoma from Normal Colon. OCT being an imaging technique, the information from this technique is conventionally analyzed qualitatively. To combine with Raman spectroscopy information, it was essential to quantify the morphological information obtained from OCT. Texture analysis was used to extract information from OCT images, which in-turn was combined with the information obtained from Raman spectroscopy. The sensitivity and specificity of the classifier was estimated using leave one out cross validation (LOOCV) method where support vector machine (SVM) was used for binary classification of the tissues. The sensitivity obtained using Raman spectroscopy and OCT individually was $89 \%$ and $78 \%$ respectively and the specificity was $77 \%$ and $74 \%$ respectively. Combining the information derived using the two techniques increased both sensitivity and specificity to $94 \%$ demonstrating that combining complementary optical information enhances diagnostic accuracy. These results demonstrate that a multimodal approach using Raman-OCT would be able to enhance the diagnostic accuracy for identifying normal and cancerous tissue types.
\end{abstract}

\section{INTRODUCTION}

It has been demonstrated by previous studies that the combination of Raman spectroscopy and optical coherence tomography (OCT) can be used as an effective multimodal tool for disease diagnosis [1-3]. There are two main challenges facing the implementation of a combined Raman-OCT system; the implementation of the hardware and the analysis of the complementary information obtained from both modalities. As previous studies have focused primarily on the implementation of the complex hardware, this study address the issue of combining the information gained from these complementary modalities. To be more specific, in this study the biochemical information of tissues from Raman spectroscopy and the morphological information of tissues from OCT are quantitatively combined. Further to this the accuracy of the classifier using both individual and multimodal information has been assessed and a clear increase in classifier accuracy has been detected when the multimodal information was included. These studies demonstrate the potential of developing multimodal systems by combining Raman spectroscopy and OCT, which in the future may be capable of performing efficient non-invasive optical biopsies.

In cancer biology neoplasia is the term used for tissues masses which grow independent and faster than the normal tissues[4]. A neoplasm, or tumour, can be further classified on the basis of its potential to harm the host as either benign or malignant (cancer). According to the Cancer Research UK statistics, there are more than 200 types of cancer. The

Biomedical Vibrational Spectroscopy VI: Advances in Research and Industry, edited by Anita Mahadevan-Jansen, Wolfgang Petrich, Proc. of SPIE Vol. 8939 89390L - (c) 2014 SPIE · CCC code: 1605-7422/14/\$18 - doi: 10.1117/12.2039855 
statistics also show that in the UK in 2010, the number of people diagnosed with cancer reached 325,000 with almost 157,000 deaths. These figures show that due to the significant number of new cases of cancer being diagnosed and the high rates of patient mortality recorded that there exists a continuing demand for intensive research to focus on understanding the origins of cancer and to provide a solution for early detection to increase the survival rate.

Neoplastic cells and tissues are identified and characterised by increases in nuclear material, cell mitoic activity and metabolic activity as well as a progressive loss of cell maturation. Studies have shown that specific changes in nucleic acid, protein, carbohydrate and lipid content which are associated with neoplastic cells can be characterised using Raman spectroscopy.[4] The most common cancer diagnosed in the UK is breast cancer, early research in this field using Raman spectroscopy by Frank et al. showed a decrease in spectral intensity associated with the diagnostic peaks corresponding to both the lipids and carotenoids for benign breast tissues[5]. Another area of high intensity research has been in the area of brain tumours, where normal tissue and neoplastic tumour samples of human origin were extensively studied[6]. Preliminary studies on colorectal carcinoma by Feld et al. have shown intense vibrational modes associated with nucleic acids in relation to carcinoma samples[7]. Research in the field of cervical cancer[8], bladder cancer[7], colon cancer[7, 9], prostate cancer[9] has also been performed and diagnostic bands have been successfully identified in relation to the biochemical makeup of these tumours.[10,11] However, improving the sensitivity (true positive rate) and specificity (true negative rate) of cancer detection, still remains to be a challenge while using Raman spectroscopy mainly due to the interference of the fluorescence background which is obtained from the sample itself.

Colorectal neoplasm is an epidemiological disease and is considered the second most common cancer in the UK[12] and the fourth most common cancer in the US [13]. The investigation of patients with suspected colorectal cancer, or the surveillance of individuals at high risk of the development of colorectal cancer, is currently performed by an endoscopic assessment of the colorectal mucosa using colonoscopy. This requires visual assessment of the mucosa, with a targeted biopsy of any abnormal areas. Both the colonoscopic assessments, and the subsequent histopathological assessment of the biopsies, are both highly subjective. The development of novel methodologies that enhance the ability to assess the colorectal mucosa would not only improve the targeting of biopsies to the most appropriate areas, but would also potentially obviate the need for tissue biopsy.

The biochemical features associated with both normal and neoplastic tissues can be distinguished due to differences in their on-going biological processes[14]. Raman spectroscopy has shown potential to detect subtle biochemical changes of various disease states within the body. However, as these changes cause alterations of biomarker concentrations at particularly low levels then a very sensitive and powerful technique is required for their detection. Clinical studies on colon cancer and pre-cancerous tissues using fluorescence spectroscopy have shown that the spectra obtained from malignant and benign abnormalities are very similar in some patients and hence hard to classify [15]. Near-IR Raman spectroscopy studies on cancerous colon tissue demonstrated the efficiency of Raman spectroscopy to identify between neoplastic and normal tissues[16, 17]. Although previous studies have demonstrated high sensitivities and specificities when discriminating between normal and adenocarcinoma colonic tissues, the Raman spectra obtained in these studies do not show a significantly different fingerprint spectrum in respect to both normal and abnormal tissue. Another crucial factor to be considered would be the occurrence of inter-patient variability in the chemical composition of colonic tissues[18]. Therefore, when considering inter-patient variability [14], the classification efficiency may be lower than the acceptable limits for using Raman spectroscopy alone as a reliable tool for disease diagnosis.

The solution to this issue would be to combine Raman spectroscopy with other optical modalities, which can provide complementary information for the tissues. Optical coherence tomography (OCT) is a powerful, non-invasive optical imaging technique that is capable of providing cross-sectional images of tissues[19, 20]. It is possible to obtain morphological information from the tissues with a resolution $\sim 10 \mu \mathrm{m}$ using OCT. While visual inspection of OCT images may be used to assess tissue morphologies, it has been demonstrated that various image-processing approaches can extract features from OCT images, which may be used to predict the biochemical state of tissues. An example of such processing techniques would be texture analysis of OCT images, which has been successful in discriminating between normal and abnormal tissue types[21,22]. The following investigation demonstrates combining quantitatively the information from Raman spectroscopy and OCT to enhance the accuracy of detection [23]. 


\section{MULTI-MODAL ANALYSIS OF TISSUE SAMPLES USING RAMAN SPECTROSCOPY AND OCT}

\subsection{The Raman spectroscopy system}

The Raman spectroscopic system used in this study was equipped with a diode laser with wavelength $785 \mathrm{~nm}$ (Sacher Lasertechnik) for Raman excitation. For detection, the system was equipped with a monochromator with a 400 lines $/ \mathrm{mm}$ grating, blazed at $850 \mathrm{~nm}$ and a deep depletion, back illuminated and thermoelectrically cooled CCD camera (Andor technologies). The laser was focused through a 40x objective (Nikon, NA 0.65 ) onto the sample in such a way that the power at the sample plane was $50 \mathrm{~mW}$ which is below the power density that can create any disruption to the morphology or chemical composition of the tissue [24]. With a $500 \mu \mathrm{m}$, confocal aperture, the confocal cylinder of the system had a base diameter of $12.5 \mu \mathrm{m}$ and a height of $10 \mu \mathrm{m}$. Tissue samples were placed on a quartz coverslip before being loaded on to the confocal Raman microscope. The back-reflection of the excitation beam was used to ensure that the confocal volume was within the sample and the position of the sample volume from the quartz coverslip was same for all the samples. The Raman spectrum from each sample was acquired with an acquisition time of $5 \mathrm{~s}$.

\subsection{Optical coherence tomography}

The OCT images were acquired utilizing a home-built Fourier domain optical coherence tomography (FDOCT) system[25]. The technique adopted in FDOCT permits the acquirement of a cross-sectional image of the sample of interest avoiding scanning of the reference arm through the depth range [26, 27], The back-reflected or back-scattered signal from the sample is acquired in a single event and the Fourier transform of the collected spectrum delivers the depth information of the sample.

The FDOCT system used in this study consisted of a fiber-coupled superluminescent diode (SLED371-HP1, Superlum Diodes, Russia) as the short-coherence light source with a bandwidth of $50 \mathrm{~nm}$ cantered at $840 \mathrm{~nm}$. Light was split between the reference arm and the sample arm by a 3-dB fiber coupler (FC850-40-50-APC, Thorlabs, NJ, USA). The reference arm was composed by a high-reflectivity gold-protected mirror (PF10-03-M01, Thorlabs, NJ, USA). Light on the sample arm was focused on the tissue of interest by a telecentric microscope objective with a working distance of 25 $\mathrm{mm}$ (LSM03-BB, Thorlabs, NJ, USA); scanning along the sample was accomplished by a sliver-coated galvanometric mirror (GVS001, Thorlabs, NJ, USA). Back-scattered light from the sample recombine with the reference light at the 3$\mathrm{dB}$ fiber coupler and the interference signal was detected by a custom spectrometer formed by a dispersive optical element (volume phase holographic grating, $1200 \mathrm{l} / \mathrm{mm}, 830 \mathrm{~nm}$, Wasatch Photonics, USA) and a line CCD detector (Aviiva EM1, part number EV71YEM1GE2014-BA9, 2048 pixels, pixel size $14 \times 28 \mu \mathrm{m}, \mathrm{e} 2 \mathrm{v}$, UK). Custom software developed using NI Labwindows (National Instruments, TX, USA) was used to control and synchronise the OCT image acquisition. All fibres used in the system were single mode for the operation wavelength (SM800-5.6-125, Thorlabs, NJ, USA). Using a high-reflectivity mirror (PF10-03-M01, Thorlabs, NJ, USA) as sample and a neutral density filter (ND40B, Thorlabs, NJ, USA) along the sample arm, the signal-to-noise ratio (SNR) of the system has been measured to be about $97 \mathrm{~dB}$ with a CCD integration time of $80 \mu \mathrm{s}$. The SNR could be improved by increasing the integration time [25].

The FDOCT system had an axial resolution of around $6.2 \mu \mathrm{m}$ limited to the light source properties and a lateral resolution of about $17 \mu \mathrm{m}$ due to the spot size on sample. The full depth range was $1.7 \mathrm{~mm}$ in air, when considering a typical colon refractive index of 1.4 [28], whilst the maximum depth range decreases to about $1.2 \mathrm{~mm}$. The lateral scan range can be set up to $5 \mathrm{~mm}$ with the galvanometric mirror with 512 lines acquired. In particular, the field of view of the images presented in this work was restricted to $1.1 \mathrm{~mm}$ in depth and $3.2 \mathrm{~mm}$ in width. The total acquisition time for a full image is about 1 second due to the CCD integration time and the data processing. The tissue sample placed on the quartz slide was scanned using the OCT system and cross sectional images were acquired from each tissue sample.

\subsection{Tissue samples}

The tissue samples used in this study were obtained from Tayside Tissue Bank (Tissue request no. TR000289). The tissue samples were snap-frozen immediately following their dissection and they were stored at $-70{ }^{\circ} \mathrm{C}$. Each sample had a dimension of approximately $2 \mathrm{~mm} \times 2 \mathrm{~mm} \times 1 \mathrm{~mm}$ as shown in Figure 1 . 


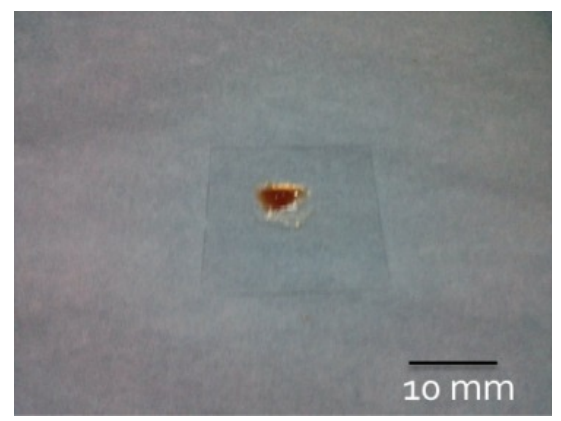

Figure 1: Typical example of colon sample used for this study. The tissue sample was thawed and placed on a quartz coverslip for Raman and OCT measurements

In this study 33 individual normal and 18 individual tumour samples were used along with 11 samples that were both normal and tumour samples (the 11 mixed samples shall hence forth be known as "mix") were analysed. For optical interrogation, the tissue sample was thawed for 5 minutes and placed on a quartz coverslip $(22 \mathrm{~mm}$ x $22 \mathrm{~mm}$, thickness $180 \mu \mathrm{m}$ ) (SPi supplies). OCT image and Raman spectrum were acquired from each sample. Further to image and spectral acquisition, the sample was fixed in formalin for histological evaluation.
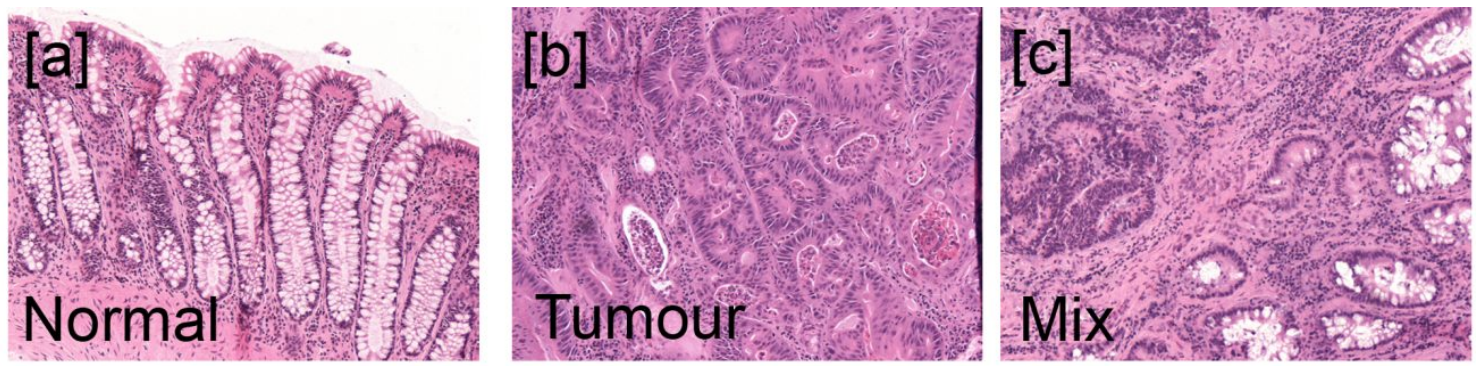

Figure 2: Representative histological images from the tissue samples used [23].

\subsection{Raman spectra analysis}

All the Raman spectra acquired from the tissue were smoothed with a Savitzky - Golay filter with smooth width 9 and degree 3 followed by baseline correction using iterative modified polynomial fitting[29]. The Raman bands that showed significant differences were estimated using a student t-test with a significance level of $10^{-3}$. A comparison of the Raman spectra obtained from two types of tissue samples are shown in Figure 3.

\subsection{Texture analysis on OCT data}

When combining a spectroscopic technique which is usually analysed quantitatively with an imaging technique that is usually analysed qualitatively, it is essential to implement a quantification algorithm for the imaging technique. It has been demonstrated in previous studies that texture analysis may be an efficient method to quantify OCT images [21, 22]. Texture is a measure of the variation of the intensity of a surface and can be quantified using properties such as smoothness, coarseness and regularity. The three principle approaches used to describe texture are statistical, structural and spectral. Statistical techniques characterise texture by the statistical properties of the grey levels of the points comprising a surface. Typically, these properties are computed from the grey level histogram or grey level co-occurrence matrix (GLCM) of the surface. The 16 texture parameters calculated from each image were the "contrast", "correlation", "energy" and "homogeneity" in four directions $\left(0^{\circ}, 45^{\circ}, 90^{\circ}\right.$ and $\left.135^{\circ}\right)$. The algorithm used for evaluating the texture parameters and avoiding the non-tissue regions of the image has been detailed in a previous study. [21] This algorithm allowed the calculation of the texture parameters independently of the morphology of the tissue surface. 


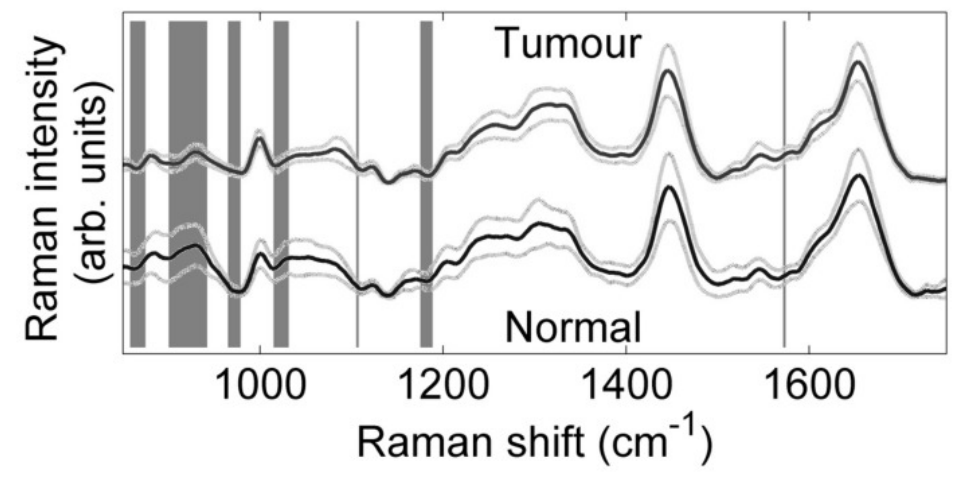

Figure 3: Comparison of Raman signature between normal and tumour tissues. The solid lines shows mean spectra of each category and the dotted lines shows the standard deviation. The vertical bar shows Raman bands that shows significance difference in student's T test with significance level $\mathrm{p}<10^{-3}[23]$.

\subsection{Data treatment and discrimination of tissue types}

PCA was used for feature selection and reduction of parameters obtained for both Raman and OCT data. A supervised discrimination algorithm using a support vector machine (SVM) with a "linear" kernel was used to perform discrimination between the two tissue types. The sensitivity and specificity of each training dataset was evaluated using leave one out cross validation (LOOCV). For the Raman and OCT dataset, the first 5 PCs were chosen to build the classifier. In the multimodal approach, the first 5 PCs of data from each modality were combined to build the classifier.

\section{RESULTS AND DISCUSSIONS}

When Raman spectroscopy data alone was considered, the sensitivity and specificity obtained was $89 \%$ and $77 \%$ respectively. It was also observed that visual examination alone for the OCT images would be too subjective in order to make a reliable diagnosis. This is also evident from the sensitivity and specificity obtained from the texture analysis of OCT data which were $78 \%$ and $74 \%$ respectively. When multi-modal information was considered, where Raman spectroscopy and OCT information were combined, $94 \%$ sensitivity and $94 \%$ specificity were obtained respectively [23]. Figure 4 shows the cluserplot of PC1 of Raman data vs. PC1 of OCT data along with the support vectors for the combined data.

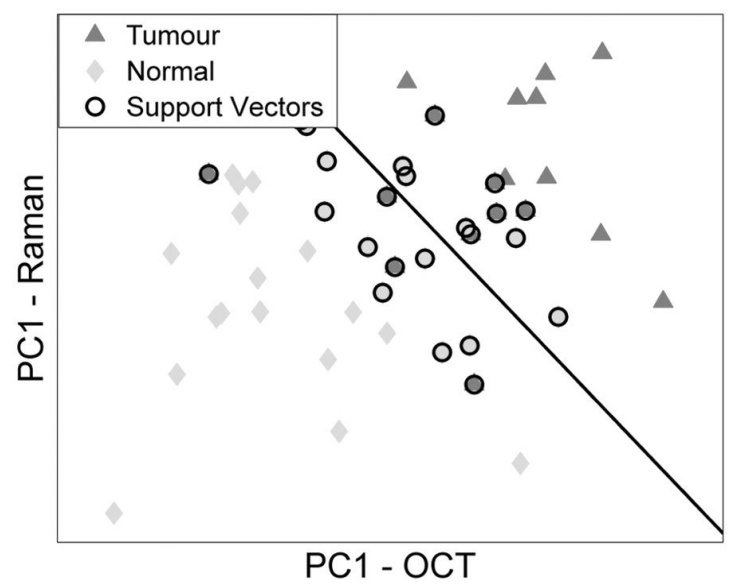

Figure 4: PC1 of Raman data vs. PC1 of OCT data clusterplot showing support vectors for the combined dataset [23]. 
Previous studies have shown that both Raman spectroscopy and OCT are powerful techniques capable of detecting cancers and pre-cancers $[15,21]$. However it is essential to assess the accuracy of these techniques so that they may be used as a reliable tool for clinical diagnostics. In that context, it is very important to consider inter-patient variability in regard to both spectral and morphological information[18]. Although previous studies demonstrated very high sensitivity and specificity in tissue discrimination [17], our observations revealed that inter-patient variability may reduce these numbers when implemented for practical applications. This is where multi-modal approaches that provide complementary information become relevant. Combination of OCT and Raman has been proposed as a potential multimodal tool for disease diagnosis $[1,2]$.

\section{CONCLUSION}

When combining Raman spectroscopy and OCT, there are two major challenges. One is combining the hardware and the other is combining the information acquired from both of these modalities. This study attempted to address the method by which information from both modalities could be combined. The main challenge to this study was the consideration that the representations of the information from these two modalities are significantly different. Raman spectra need to be quantified to obtain useful inferences, while conventionally the OCT images are visually analysed. The approach taken in this study to combine these two modalities was to quantify the OCT images using texture analysis. The texture information obtained from OCT could be readily combined with the spectral information from Raman spectroscopy. The sensitivity and specificity of the classifier saw a greater than $10 \%$ increase when multi-modal information was used for tissue discrimination as opposed to using single modality. This shows the potential of combining Raman spectroscopy and OCT for implementing reliable, optical diagnostic systems. While colon tissue samples were used in this study, it would be possible to extend such approaches for discriminating other tissue types as well.

\section{ACKNOWLEDGEMENTS}

We thank the UK EPSRC for funding, the CR-UK/EPSRC/MRC/DoH (England) imaging programme, the European Union project FAMOS (FP7 ICT, contract no. 317744) and the European Union project IIIOS (FP7/2007-2013, contract no. 238802). We thank Tayside Tissue Bank for providing us with the tissue samples under request number TR000289. K.D. is a Royal Society-Wolfson Merit Award Holder.

\section{REFERENCES}

[1] C. A. Patil, N. Bosschaart, M. D. Keller et al., "Combined Raman spectroscopy and optical coherence tomography device for tissue characterization," Optics Letters, 33(10), 1135-1137 (2008).

[2] J. W. Evans, R. J. Zawadzki, R. Liu et al., "Optical coherence tomography and Raman spectroscopy of the exvivo retina," Journal of Biophotonics, 2(6-7), 398-406 (2009).

[3] K. M. Khan, H. Krishna, S. K. Majumder et al., "Depth-sensitive Raman spectroscopy combined with optical coherence tomography for layered tissue analysis," Journal of Biophotonics, n/a-n/a (2013).

[4] A. Mahadevan-Jansen, and R. R. Richards-Kortum, "Raman spectroscopy for the detection of cancers and precancers," Journal of Biomedical Optics, 1(1), 31-70 (1996).

[5] C. J. Frank, D. C. B. Redd, T. S. Gansler et al., "Characterization of human breast biopsy specimens with nearIR Raman spectroscopy,” Analytical Chemistry, 66(3), 319-326 (1994).

[6] A. Mizuno, H. Kitajima, K. Kawauchi et al., "Near-infrared Fourier transform Raman spectroscopic study of human brain tissues and tumours," Journal of Raman Spectroscopy, 25(1), 25-29 (1994).

[7] M. S. Feld, R. Manoharan, J. Salenius et al., "Detection and characterization of human tissue lesions with nearinfrared Raman spectroscopy,” 99-104 (1995).

[8] C. H. Liu, B. B. Das, W. L. S. Glassman et al., "Raman, fluorescence, and time-resolved light scattering as optical diagnostic techniques to separate diseased and normal biomedical media," Journal of Photochemistry and Photobiology B: Biology, 16(2), 187-209 (1992). 
[9] N. Stone, C. Kendall, J. Smith et al., "Raman spectroscopy for identification of epithelial cancers," Faraday Discussions, 126(0), 141-157 (2004).

[10] A. Nijssen, S. Koljenovic, T. C. Bakker Schut et al., "Towards oncological application of Raman spectroscopy," Journal of Biophotonics, 2(1-2), 29-36 (2009).

[11] A. Nijssen, T. C. Bakker Schut, F. Heule et al., "Discriminating Basal Cell Carcinoma from its Surrounding Tissue by Raman Spectroscopy," 119(1), 64-69 (2002).

[12] C. R. UK, [Bowel cancer Key Facts] Cancer Research UK, (2013).

[13] C. Scalfi-Happ, M. Udart, C. Hauser et al., "Investigation of lipid bodies in a colon carcinoma cell line by confocal Raman microscopy,” Medical Laser Application, 26(4), 152-157 (2011).

[14] N. Stone, C. Kendall, J. Smith et al., "Raman spectroscopy for identification of epithelial cancers," Faraday Discussions, 126, 141-157 (2004).

[15] A. Mahadevan-Jansen, and R. R. Richards-Kortum, "Raman spectroscopy for the detection of cancers and precancers," J Biomed Opt, 1(1), 31-70 (1996).

[16] M. S. Feld, R. Manoharan, J. Salenius et al., "Detection and characterization of human tissue lesions with near infrared Raman spectroscopy," Advances in Fluorescence Sensing Technology Ii, 2388, 99-104 (1995).

[17] E. Widjaja, W. Zheng, and Z. W. Huang, "Classification of colonic tissues using near-infrared Raman spectroscopy and support vector machines,” International Journal of Oncology, 32(3), 653-662 (2008).

[18] P. O. Andrade, R. A. Bitar, K. Yassoyama et al., "Study of normal colorectal tissue by FT-Raman spectroscopy," Anal Bioanal Chem, 387(5), 1643-8 (2007).

[19] S. A. Boppart, "Optical coherence tomography - Principles applications and advances," Minerva Biotecnologica, 16(4), 211-237 (2004).

[20] A. F. Fercher, W. Drexler, C. K. Hitzenberger et al., "Optical coherence tomography - principles and applications," Reports on Progress in Physics, 66(2), 239-303 (2003).

[21] M. Bhattacharjee, P. C. Ashok, K. D. Rao et al., "Binary tissue classification studies on resected human breast tissues using optical coherence tomography images," Journal of Innovative Optical Health Sciences, 4(1), 59-66 (2011).

[22] K. W. Gossage, T. S. Tkaczyk, J. J. Rodriguez et al., "Texture analysis of optical coherence tomography images: feasibility for tissue classification,”J Biomed Opt, 8(3), 570-5 (2003).

[23] P. C. Ashok, B. B. Praveen, N. Bellini et al., "Multi-modal approach using Raman spectroscopy and optical coherence tomography for the discrimination of colonic adenocarcinoma from normal colon," Biomed. Opt. Express, 4(10), 2179-2186 (2013).

[24] M. Larraona-Puy, A. Ghita, A. Zoladek et al., "Development of Raman microspectroscopy for automated detection and imaging of basal cell carcinoma," Journal of Biomedical Optics, 14(5), (2009).

[25] N. Krstajic, C. T. A. Brown, K. Dholakia et al., "Tissue surface as the reference arm in Fourier domain optical coherence tomography," Journal of Biomedical Optics, 17(7), 071305 (2012).

[26] A. F. Fercher, C. K. Hitzenberger, G. Kamp et al., "Measurement of intraocular distances by backscattering spectral interferometry," Optics Communications, 117(1-2), 43-48 (1995).

[27] R. Leitgeb, C. Hitzenberger, and A. Fercher, "Performance of fourier domain vs. time domain optical coherence tomography," Opt Express, 11(8), 889-94 (2003).

[28] G. Zonios, L. T. Perelman, V. M. Backman et al., "Diffuse reflectance spectroscopy of human adenomatous colon polyps in vivo," Applied Optics, 38(31), 6628-6637 (1999).

[29] C. A. Lieber, and A. Mahadevan-Jansen, "Automated method for subtraction of fluorescence from biological Raman spectra,” Applied Spectroscopy, 57(11), 1363-1367 (2003). 